The result given above for unrestricted $x$ suggests my second proof, which is considerably simpler. From the asymptotic expansion of the Gamma-function it follows that the function

$$
-\frac{\Gamma(x) \Gamma\left(-x-\frac{1}{2}\right)}{8 \Gamma\left(\frac{3}{2}+x\right) \Gamma(1-x)}
$$

satisfies the conditions (Cf. Modern Analysis, $\S 7 \cdot 4$ ) which admit of its being expressed as a sum of partial fractions. Since the residues of the function at the poles $x=-n, x=n-\frac{1}{2}$ are respectively

we obtain anew the result

$$
\frac{1}{2} A_{n}, \quad-\frac{1}{2} A_{n},
$$

$$
-\frac{\Gamma(x) \Gamma\left(-x-\frac{1}{2}\right)}{8 \Gamma\left(\frac{3}{2}+x\right) \Gamma(1-x)}=\sum_{n=0}^{\infty} \frac{A_{n}}{2 x+2 n}-\sum_{n=0}^{\infty} \frac{A_{n}}{2 x-2 n+1}
$$

for unrestricted $x$.

It is, of course, possible to write down any number of generalisations of this result, the simplest perhaps being the expression for

$$
\frac{\Gamma(x) \Gamma(-x-a)}{\Gamma(1+a+x) \Gamma(1-x)}
$$

as a sum of partial fractions, where $a$ is a suitably restricted constant.

\title{
On the configuration known as a double-six of lines
}

By H. W. Richmond, King's College, Cambridge.

In geometry of three dimensions it is well known that, when two quadrics $Q_{1}, Q_{2}$ are given, if one set of four points exists having the properties that each point lies on $Q_{1}$, and each two points are conjugate with respect to $Q_{2}$, an infinity of such sets of points can be found. The quadrics $Q_{1}, Q_{2}$ stand in a special relation to one another ${ }^{1}$, expressed by the vanishing of the coefficient of $\lambda$ in the discriminant of $Q_{2}+\lambda Q_{1}$, an invariant of $Q_{1}, Q_{2}$. Two quadrics $Q_{1}, Q_{2}$ are thus related if the equation of $Q_{1}$ contains no squares

1 See Salmon, Analytic Geometry of Three Dimensions, Rogers' revised edition, Vol. 1, p. 204 ; or Sommerville, Analytical Geometry of Three Dimensions, p. 309. 
of the coordinates, and that of $Q_{2}$ contains no products of two coordinates; for then the vertices of the tetrahedron of reference form such a set of four points.

Even better known are the analogous properties in plane geometry concerning sets of three points and two conics: they need not be quoted. Similar results hold when more than four homogeneous variables are used; they may be enunciated as theorems in algebra, or in geometry of more than three dimensions. With six homogeneous coordinates, if in the first paragraph we replace "four" by "six," and the word "tetrahedron" by "simplex," we have a theorem of geometry of five dimensions.

But under certain conditions the six coordinates lend themselves to a simple interpretation in three-dimensional space. Points being represented by homogeneous coordinates $\left(x_{0}, x_{1}, x_{2}, x_{3}\right)$, Plücker represented a line by six homogeneous coordinates $p_{01}, p_{02}, p_{03}, p_{23}$, $p_{31}, p_{12}$ satisfying a quadratic relation

$$
p_{01} p_{23}+p_{02} p_{31}+p_{03} p_{12} \equiv 0:
$$

since this contains no squared terms, it will serve for $Q_{1}$. Again, if $S$ is any quadric surface in the space whose equation is expressed as

$$
a_{0} x_{0}^{2}+a_{1} x_{1}^{2}+a_{2} x_{2}^{2}+a_{3} x_{3}^{2}=0,
$$

the condition that a line should touch $S$ is

$$
\begin{aligned}
& a_{0} a_{1} p_{01}^{2}+a_{0} a_{2} p_{02}^{2}+a_{0} a_{3} p_{03}^{2}+a_{2} a_{3} p_{23}^{2}+a_{3} a_{1} p_{31}^{2}+a_{1} a_{2} p_{12}^{2}=0, \\
& \text { or } \quad \sum a_{m} a_{n} p_{m n}^{2}=0:
\end{aligned}
$$

which, containing only squares of the coordinates $(p)$, will serve for $Q_{2}$. The condition

$$
\Sigma a_{m} a_{n} p_{m n} p_{m n}^{\prime}=0,
$$

(which asserts that two sets of six coordinates $p_{m n}$ and $p_{m n}^{\prime}$ are conjugate with respect to $Q_{2}$ ) bears the interpretation that either line meets the polar of the other with respect to $S$. Hence we have the theorem.

In connection with any quadric surface in space of three dimensions, there exist sets of six lines having the property that any one of the lines intersects the polar line of any other.

The quadric being given, a set of six lines may be built up as follows:- the first line $\left(a_{1}\right)$ is chosen at random; its polar line $\left(b_{1}\right)$ is then known. The second line $\left(a_{2}\right)$ may be any line that meets $b_{1}$; 
$\mathbf{v i}$

its polar line $\left(b_{2}\right)$, which must meet $a_{1}$, is then known. For $a_{3}$ choose any line that meets $b_{1}$ and $b_{2}$; its polar line $\left(b_{3}\right)$ will meet $a_{1}$ and $a_{2}$ and will be known. For $a_{4}$ choose any line that meets $b_{1}, b_{2}, b_{3}$; its polar line $\left(b_{4}\right)$ will meet $a_{1}, a_{2}, a_{3}$ and will be known. But at this point, since only two lines meet $b_{1}, b_{2}, b_{3}, b_{4}$, one of them must be $a_{\tilde{s}}$ and the other $a_{6}$; their polars $b_{5}$ and $b_{6}$ are the two lines which meet $a_{1}, a_{2}, a_{3}, a_{4}$ : and at this point, since all but one of the conditions binding the lines (a) have been satisfied, the force of the invariant relation between $Q_{1}$ and $Q_{2}$ becomes effective and proves the last condition, viz. that $a_{5}$ meets the polar line of $a_{6}$ and vice versa. The twelve lines $a$ and $b$ thus constitute a double-six of lines. Moreover any double-six of lines may be thus obtained.

\section{The evaluation of certain continued fractions}

By C. G. Darwin, University of Cambridge.

1. If the approximate numerical value of $e$ is expressed as a continued fraction the result is

$$
e=2+\frac{1}{1}+\frac{1}{2}+\frac{1}{1}+\frac{1}{1}+\frac{1}{4}+\frac{1}{1}+\frac{1}{1}+\frac{1}{6}+\frac{1}{1} \ldots
$$

and it was in finding the proof that the sequence extends correctly to infinity that the following work was done. First the continued fraction may be simplified by setting down the difference equations for numerator and denominator as usual, and eliminating two out of every successive three equations. A difference equation is thus formed between the first, fourth, seventh, tenth .... convergents (counting the first as $2+\frac{1}{1}$ ), and this equation will generate another continued fraction. After a little rearrangement of the first two members it appears that (1) implies

$$
\frac{e-1}{e+1}=\frac{1}{2}+\frac{1}{6}+\frac{1}{10}+\cdots
$$

2. We therefore consider the continued fraction

$$
F\left(\begin{array}{ll}
\alpha & \beta \\
\gamma & \delta
\end{array}\right)=\frac{a}{\gamma}+\frac{a+\beta}{\gamma+\delta}+\frac{a+2 \beta}{\gamma+2 \delta}+\cdots
$$

